\title{
Multiphase CFD Modelling of Single-Use-Technology Bioreactors for Industrial Biotechnology Applications
}

\author{
Richard Maltby ${ }^{1,2}$, William Lewis ${ }^{1}$, Stephen Wright ${ }^{3}$, Alex Smith ${ }^{3}$, John Chew $^{1}$ \\ ${ }^{1}$ Department of Chemical Engineering, University of Bath \\ Bath, BA2 7AY, UK \\ ${ }^{2}$ Centre for Sustainable Chemical Technologies, University of Bath \\ Bath, BA2 7AY, UK \\ ${ }^{3}$ Centre for Process Innovation, The Wilton Centre \\ Teesside, TS10 4RF, UK \\ R.P.Maltby@bath.ac.uk; Y.M.Chew@bath.ac.uk
}

\begin{abstract}
Multiphase CFD modelling has been applied to a cubic geometry single-use-technology (SUT) bioreactor as a tool during the design process. A non-cylindrical tank geometry is employed to make the platform more appealing to wider industrial biotechnology applications in terms of cost and simplicity, with mass transfer investigated in terms of $\mathrm{k}_{\mathrm{L}}$ a values. Five models for the mass transfer coefficient $\mathrm{k}_{\mathrm{L}}$ are compared for a range of different conditions. The effect of varying the impeller rotational speed is investigated, with an optimum value of 400 RPM identified. Increasing the aeration rate from 0.0675 to $0.1 \mathrm{vvm}$ has also been shown to increase the average $\mathrm{k}_{\mathrm{L}}$ a values within the vessel by approximately $50 \%$. An existing 1,000 L vessel is modelled, as well as a theoretical $200 \mathrm{~L}$ alternative, which showed much improved mass transfer for the same inlet gas velocity. Turbulence is modelled using the k- $\varepsilon$ model and impeller motion is modelled using the moving reference frame method.
\end{abstract}

Keywords: CFD, mixing, mass transfer, Single-Use-Technologies, turbulence

\section{Nomenclature}

$\rho \quad$ density $\left(\mathrm{kgm}^{-3}\right)$

$\mathrm{u} \quad$ velocity $\left(\mathrm{ms}^{-1}\right)$

$\mathrm{t}$ time (s)

$\mathrm{x} \quad$ distance $(\mathrm{m})$

$\tau \quad$ shear stress tensor

$\mathrm{F} \quad$ body forces $(\mathrm{N})$

$\mathrm{k} \quad$ turbulent kinetic energy $\left(\mathrm{m}^{2} \mathrm{~s}^{-2}\right)$

$\mu \quad$ dynamic viscosity (Pa.s)

$\varepsilon \quad$ eddy dissipation rate $\left(\mathrm{m}^{2} \mathrm{~s}^{-3}\right)$

$\mathrm{C}_{\varepsilon} \sigma_{\mathrm{k}, \varepsilon} \mathrm{k}-\varepsilon$ closure coefficients (-)

$\mathrm{N}$ mass transfer rate $\left(\mathrm{molm}^{-3} \mathrm{~s}^{-1}\right)$
$\mathrm{P} \quad$ absolute pressure $(\mathrm{Pa})$

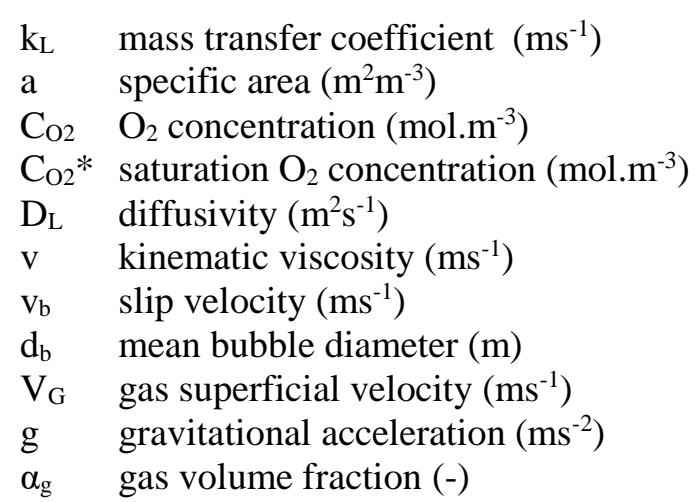

\section{Introduction}

Single-use-technologies are a category of disposable bioprocessing components which have increased in popularity in the biopharmaceutical industry in recent years. The first instance of single-use components being used in the biotechnology field occurred during the 1960's, when laboratory-scale glassware and bioreactors were replaced by disposable plastic alternatives [1]. However, it was not until the late 1990's that SUT was applied on an industrially significant scale [1], with the introduction of a series of wave-type bioreactors with a working volume of up to $500 \mathrm{~L}$ [2]. Mixing in these devices is achieved through the gentle rocking of a partially fluid-filled bag, which lends itself well to biopharmaceutical cultures of mammalian cells due to their low-shear requirements and low oxygen demands. 
Single-use-technologies have also been applied to stirred bioreactors up to the 2,000 L scale [2], with several manufacturers offering commercial stirred SUT bioreactors of varying design and capacity, as summarised by Lopes [3]. Like their wave-type counterparts, the main area of application has been the production of vaccines by mammalian $[1,3]($ and less commonly plant [4]) cells. The wider field of industrial biotechnology has been largely untouched by growth in popularity of SUT, where lower-value products are produced in large volumes and with high cell densities. Aeration and mixing requirements are therefore high, and not easily achieved in currently available SUT designs. There is, therefore, an opportunity for a low-cost SUT bioreactor to be developed for the wider bioproduction industry, with the effective design of mixing and aeration required to support large-scale productions.

The benefits of employing SUT bioreactors, and therefore the drivers behind their adoption, can be related to a combination of production flexibility, cost and environmental impact. Firstly, the use of pre-sterilised bags means that the time required in between batches is reduced from 8-10 hours for traditional bioreactors to between 1 and 2 hours [2]. Furthermore, it gives the flexibility to change between different products much more easily, with traditional sterilisation and validation for such process changes taking up to three weeks [2]. The risk of cross-contamination from manufacturing different products is also virtually eliminated [5]. Reduced capital and operating costs for hybrid (part-SUT) and fully SUT facilities are reported versus traditional stainless steel facilities for the production of viral vaccines [3]. Significant savings are made in most areas of build and operation, including utilities and labour, with the notable exception of consumables (replacing the disposable SUT items) and disposal of solid plastic waste. Despite the large amounts of plastic waste SUT operations can expect to incur, the overall environmental impact of the process is reduced when the whole lifecycle is considered [6]. Energy consumption is reduced by approximately $50 \%$ by removing the need for large amounts of steam used for cleaning and sterilisation in-place [3], as well as eliminating the need for harsh chemicals in the sterilisation process. Further guidance on best practice for the disposal of SUT components is available from industry [7].

In this work, CFD modelling has been applied during the design process of a commercial SUT for the industrial biotechnology sector to provide support in selecting the optimum operating conditions, as well as providing an efficient assessment of proposed design decisions and alterations. Specifically, the impeller speed and gas flow rate have been varied, and changes in local and volume-averaged $\mathrm{k}_{\mathrm{L}} \mathrm{a}$ values are used as the main criterion on which the impact is assessed. A 200 L variation of existing 1,000 L cubic geometry has also been investigated, with this shape preferred for the lower scale SUT application due to the ease of production and installation, as well as reduced cost and complexity of manufacture. A magnetic stirrer is also used for reduced complexity and to improve bag integrity.

\section{Numerical Modelling}

\subsection{Computation Domain}

Two different domains are considered in this work, with operating volumes of 1,000 L (Figure 1a) and $200 \mathrm{~L}$ (Figure 1b). The modelling domain of the 1,000 L vessel is half that of the actual geometry to reduce computational expense. Further reductions are not possible as there is only a single symmetry plane due to the location of the spargers in two circular rings, an inner ring consisting of eight spargers and an outer ring with six equally spaced spargers located around the centrally mounted impeller (Figure 1c). The tank geometry consists of a $1 \times 1 \times 1 \mathrm{~m}$ cube. Spargers are modelled as highly porous active surfaces located around a section of the outer cylinder, which is modelled as the gas inlet. The impeller is based on a 163 $\mathrm{mm}$ diameter disposable magnetically-driven design, as shown in Figure 1c, and is located on the floor of the tank. This is used for the SUT as it does not require any moving parts to penetrate the bag, unlike traditional shaft-driven impellers. Impeller motion is modelled using the moving reference frame (MRF) method, where the mesh remains rigid and a body force is applied to the fluid in the MRF region to simulate impeller motion. The MRF region is defined as a cylinder which extends between the impeller tip and the inner sparger ring, as shown in Figure 1.

The $200 \mathrm{~L}$ geometry is based on a proposed design for improving the mass transfer in the SUT vessel by reducing the tank size. It uses the same impeller and inner sparger ring designs, however there are now only four outer spargers, located equidistant between the impeller tip and corner of the vessel. This means that there are now two symmetry planes, one along each impeller blade, and therefore only a quarter of the physical geometry is modelled, as shown in Figure 1b. The base is a simple $0.616 \times 0.616 \mathrm{~m}$ square, with the fluid level also set to $0.616 \mathrm{~m}$ to maintain the cubic geometry, giving a modelled working volume of $233 \mathrm{~L}$. Meshing for each model is achieved using a fully unstructured tetrahedral mesh, owing to the complex geometry formed around the MRF region and the sparger heads. The mesh element size has been manually optimised to give a high resolution in the impeller region whilst reducing the overall number of elements as far as possible. 


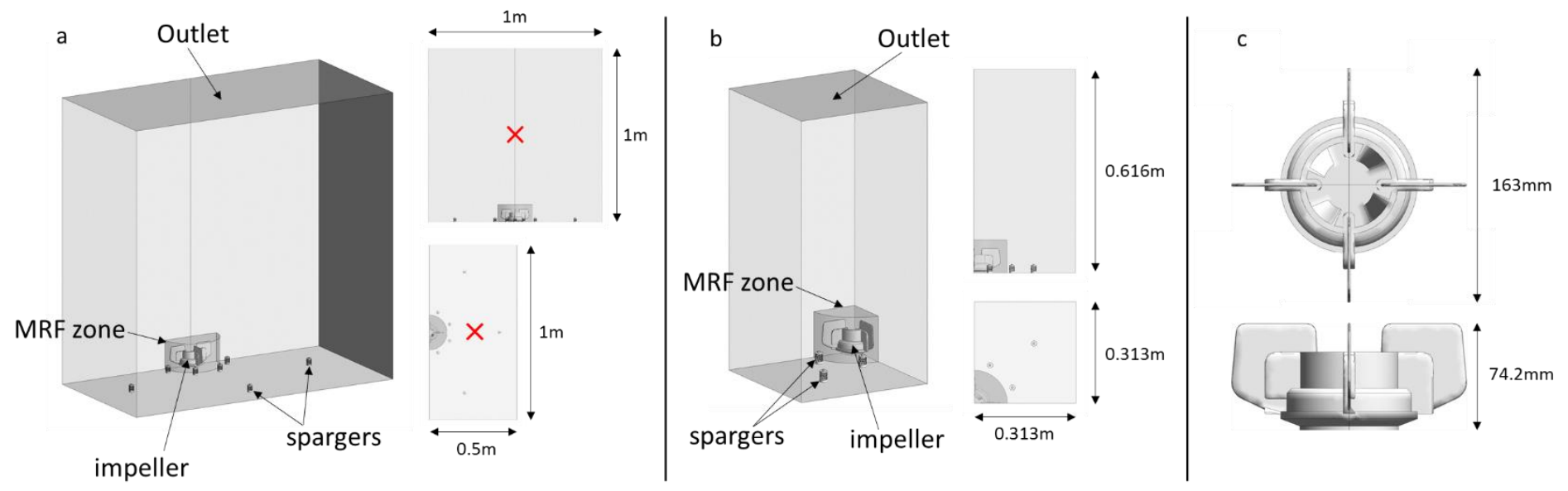

Fig. 1: 3D modelling domains for a) 1,000 L geometry, b) 200 L geometry, and c) impeller design.

The cross indicates the location of dissolved oxygen measurements in the $1000 \mathrm{~L}$ tank.

\subsection{Governing Equations}

CFD modelling was performed using the commercial CFD software ANSYS CFX-15. This uses a finite volume discretisation method to approximate the continuity (equation 1) and Navier-Stokes (equation 2) equations (in tensor notation) for an incompressible Newtonian fluid [8]. All symbols used are defined in Nomenclature.

$$
\begin{gathered}
\frac{\partial \rho}{\partial t}+\frac{\partial \rho u_{j}}{d x_{j}}=0 \\
\rho \frac{\partial u_{i}}{\partial t}+\rho u_{j} \frac{\partial u_{i}}{\partial x_{j}}=\frac{\partial P}{\partial x_{i}}+\frac{\partial \tau_{i j}}{\partial x_{j}}+F_{i}
\end{gathered}
$$

Turbulence is modelled using the k- $\varepsilon$ model, which is a two equation model described by equations 3 and 4 [8]. This is applied in two phases using the dispersed phase zero equation model, which relates the dispersed and continuous phase kinematic eddy viscosities via a term called the turbulent Prandtl number, as described in the solver documentation [9].

$$
\begin{aligned}
& \rho \frac{\partial k}{\partial t}+\rho\left\langle u_{j}\right\rangle \frac{\partial k}{\partial x_{j}}=\mu_{T}\left[\left(\frac{\partial\left\langle u_{i}\right\rangle}{\partial x_{j}}+\frac{\partial\left\langle u_{j}\right\rangle}{\partial x_{i}}\right) \frac{\partial u_{i}}{\partial x_{j}}\right]-\rho \varepsilon+\frac{\partial}{\partial x_{j}}\left[\left(\mu+\frac{\mu_{T}}{\sigma_{k}}\right) \frac{\partial k}{\partial x_{j}}\right] \\
& \rho \frac{\partial \varepsilon}{\partial t}+\rho\left\langle u_{j}\right\rangle \frac{\partial \varepsilon}{\partial x_{j}}=C_{\varepsilon 1} \mu_{T} \frac{\varepsilon}{k}\left[\left(\frac{\partial\left\langle u_{i}\right\rangle}{\partial x_{j}}+\frac{\partial\left\langle u_{j}\right\rangle}{\partial x_{i}}\right) \frac{\partial\left\langle u_{i}\right\rangle}{\partial x_{j}}\right]-C_{\varepsilon 2} \rho \frac{\varepsilon^{2}}{k}+\frac{\partial}{\partial x_{j}}\left[\left(\mu+\frac{\mu_{T}}{\sigma_{\varepsilon}}\right) \frac{\partial \varepsilon}{\partial x_{j}}\right]
\end{aligned}
$$

The values of the closure coefficients used in these equations are as follows; $C_{\varepsilon 1}=1.45, C_{\varepsilon 2}=1.9, \sigma_{k}=1.0, \sigma_{\varepsilon}=1.3$.

Buoyancy is modelled based on the density difference between the two fluids, since heat transfer is not considered for this model. An additional drag force is also applied for multiphase modelling, which represents the force exerted by the relative motion of the two fluids. The Schiller Naumann correlation is used to calculate the drag coefficient associated with this force, with the assumption that the bubble size produced by the porous sparger surface is small enough to form only spherical bubbles. To save on computational expense, other interphase momentum transfer phenomena are neglected due to the reported dominance of the drag force [10].

Boundary conditions for the outlet at the top of the tank are modelled as an opening, allowing the escape of gas from the modelled domain, with a volume fraction of 1 for the continuous phase also specified. The no slip boundary condition is applied to all solid walls, with isothermal operation assumed. The fluids modelled are water for the continuous phase and air for the dispersed phase, with physical properties taken at $25^{\circ} \mathrm{C}$. For this model, the bubble size at the inlet is assumed to be 
a constant $1 \mathrm{~mm}$ diameter, with an inlet gas fraction of 1 at the active surface of the spargers. Symmetry planes are modelled as periodic boundaries.

\subsection{Mass Transfer Models}

Mass transfer in aerated bioreactors is often reported in terms of $\mathrm{k}_{\mathrm{L}} \mathrm{a}$ values, which can be easily determined for laboratory-based and industrial vessels by tracking dissolved oxygen levels over time, known as the dynamic method of $\mathrm{k}_{\mathrm{L}} \mathrm{a}$ estimation [11], however this is not always available during the design phase for novel bioreactors. A number of models for determining $\mathrm{k}_{\mathrm{L}}$, the mass transfer coefficient of oxygen into the liquid phase, are proposed in literature, as summarised by Kulkarni [12]. The models can subsequently be put to use as a tool to either predict in advance the mass transfer or better still, design a system to produce a specific outcome, thus reducing the amount of trial and error work. This is particularly useful in refining, down- and up-scaling of the mixing process. Of these models, only some have the necessary parameters to be calculated from the results of a CFD model. Five models which have been identified as being appropriate for use alongside CFD modelling are discussed in Table 1.

Table 1: Models used to calculate the transfer coefficient [12,13].

\begin{tabular}{|c|c|c|}
\hline Mass Transfer Model & $\begin{array}{c}\text { Eqn. } \\
\#\end{array}$ & Description \\
\hline $\mathrm{k}_{L \text { penetration }}=\frac{2}{\sqrt{\pi}} \sqrt{D_{L} \sqrt{\frac{\varepsilon}{v}}}$ & $(5)$ & $\begin{array}{l}\text { The penetration model is based on Higbie's penetration theory of } \\
\text { interface transfer [14]. It is assumed that the mass transfer occurs largely } \\
\text { due to the effect of small eddies, so that the Kolmogorov Length Scale is } \\
\text { used to describe the contact time. The model is therefore dependent on } \\
\text { the eddy dissipation rate }(\varepsilon) \text { which is a key parameter in the k- } \varepsilon \\
\text { turbulence model. }\end{array}$ \\
\hline $\mathrm{k}_{L \text { eddy cell }}=0.4 \sqrt{D_{L} \sqrt{\frac{\varepsilon}{v}}}$ & (6) & $\begin{array}{l}\text { The eddy cell model was proposed by Lamont and Scott [15] and has a } \\
\text { very similar form to the penetration theory, however it is based on the } \\
\text { surface renewal model. Here, it is assumed that the surface renewal rate } \\
\text { is calculated using the Kolmogorov Scale model due to the influence of } \\
\text { small eddies in mass transfer. }\end{array}$ \\
\hline $\mathrm{k}_{L \text { slip velocity }}=\frac{2}{\sqrt{\pi}} \sqrt{\frac{D_{L} v_{b}}{d_{b}}}$ & (7) & $\begin{array}{l}\text { The slip velocity model is based on the difference in velocity between } \\
\text { the gas and liquid phases. As with the eddy cell model, the slip velocity } \\
\text { model uses surface renewal theory as a starting point. However, in this } \\
\text { case the renewal of fluid at the surface was assumed to be due to the } \\
\text { relative motion of the bulk gas and liquid phases, as referenced by } \\
\text { Ranganathan and Sivaraman [13]. }\end{array}$ \\
\hline $\mathrm{k}_{\text {L rigid }}=0.6\left(\frac{v_{b}}{d_{b}}\right)^{1 / 2}\left(D_{L}\right)^{3 / 2} v^{-1 / 6}$ & (8) & $\begin{array}{l}\text { For the rigid model, Alves [16] proposed that if a bubble was sufficiently } \\
\text { rigid, } \mathrm{k}_{\mathrm{L}} \text { could be described based upon the Frossling equation for } \\
\text { laminar boundary layers, with an experimentally derived constant of } 0.6 \text {. } \\
\text { This simplification is therefore only applicable to smaller bubbles, which } \\
\text { maintain a more spherical shape. }\end{array}$ \\
\hline $\mathrm{k}_{L s r s}=\frac{2}{\sqrt{\pi}} \sqrt{D_{L} \sqrt{\frac{V_{G} g}{v}}}$ & (9) & $\begin{array}{l}\text { The final model discussed is known as the surface renewal stretch } \\
\text { model, as proposed by Jajuee [17]. It is different to the other models } \\
\text { proposed as it combines the continuity equation with aspects of surface } \\
\text { renewal theory and penetration theory for surface stretch. The model has } \\
\text { been correlated against experimental data, with a high degree of accuracy } \\
\text { claimed [17]. The equation uses the superficial velocity of the gas phase, } \\
\text { which can be found from the CFD model }\end{array}$ \\
\hline
\end{tabular}

$\mathrm{k}_{\mathrm{L}} \mathrm{a}$ values are calculated for each model by multiplying the mass transfer coefficient by the specific area, a. This is the area of the interface between the gas and liquid phases and can be calculated for spherical bubbles using equation 10 . For this model, a constant bubble size (the same as the inlet condition) is assumed throughout the tank. 


$$
\mathrm{a}=\frac{6 \alpha_{G}}{d_{b}}
$$

\section{Results and Discussions}

\subsection{Effect of Varying Impeller Speed}

A comparison of the different models for $\mathrm{k}_{\mathrm{L}}$ discussed in Table 1 has been made for varying stirrer speeds. The volumeaveraged $\mathrm{k}_{\mathrm{L}}$ a values for the 1,000 L tank are compared in Figure 2. All of the models show an increase in mean $\mathrm{k}_{\mathrm{L}} \mathrm{a}$ value with impeller speed up to 400 RPM, above which the models based on eddy dissipation begin to level off whereas the others continue to increase. The range of values obtained for $\mathrm{k}_{\mathrm{L}}$ a is quite large, although all are of a similar order of magnitude to typical industrial bioreactors $\left(72-900 \mathrm{hr}^{-1},[11]\right)$, albeit at the lower end due to the limitations in geometry and mixing imposed by the SUT concept. Higher stirrer speeds will cause more shear and undesirable conditions for cell culture, and increasing the stirrer speed above 500 RPM may also cause a lifting effect in the reactor.

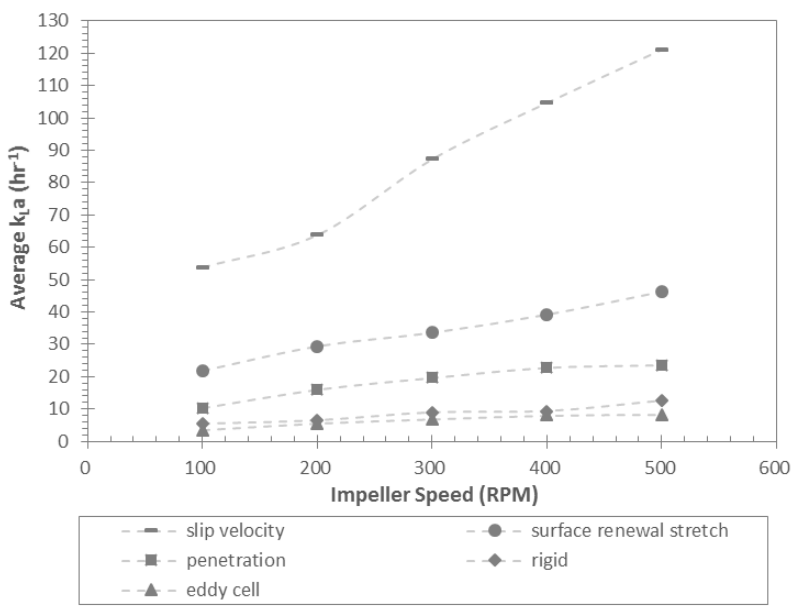

Fig. 2: Comparison of $\mathrm{k}_{\mathrm{L}}$ a values from different mass transfer models at different impeller rotational speeds.

Analysis of the gas dispersion within the vessel (Figure 3) shows that there is a regime change between the 200 and 300 RPM solutions. At lower impeller speeds, the radial dispersion is not great enough to overcome the buoyancy of the dispersed gas within the fluid, leading to the formation of a gas column rising through the centre of the tank. In contrast, impeller speeds of 300 RPM and above give a much more even distribution of the gas phase, with the radial action of the impeller now dispersing gas along the base of the tank and into the corners of the vessel. Some recirculation can be seen in Figure 3d, with the liquid velocity vectors showing fluid in the lower third of the vessel being drawn back towards the impeller, and an upwards flow of liquid which is much less focussed towards the centre of the vessel. Note that the maximum $\mathrm{k}_{\mathrm{L}}$ a in Figure 3 has been limited to $100 \mathrm{hr}^{-1}$ to aid comparison between conditions.

The $\mathrm{k}_{\mathrm{L}}$ a values modelled in the 1,000 L tank have been compared to a single $\mathrm{k}_{\mathrm{L}}$ a measurement taken in the existing tank of the same geometry at the location indicated by the cross in Figure 1a. The experimental $\mathrm{k}_{\mathrm{L}} \mathrm{a}$ value was calculated using the dynamic method of measurement, which tracks dissolved oxygen concentration over time at a single point rather than the instantaneous $\mathrm{k}_{\mathrm{L}}$ a values presented from the model. The eddy cell model provided the best fit to the data collected at this point at $0.1 \mathrm{vvm}$ and $400 \mathrm{RPM}$. This choice of model is supported by the work of Ranganathan and Sivaraman [13] which concluded that the eddy cell model provided the best fit to gas holdup values from experimental work [16] when modelling a multi-impeller vessel of identical dimensions. The model presented currently uses a fixed bubble size. In reality, significant bubble coalescence is expected away from the impeller, which will have an impact on the flow patterns in the tank due to the higher rise velocity of large bubbles. From Figure 2, the chosen model suggests that a stirrer speed of 500 RPM offers no additional benefit in terms of mass transfer, and it is therefore recommended that this geometry is run with an optimum impeller speed of $400 \mathrm{RPM}$. 


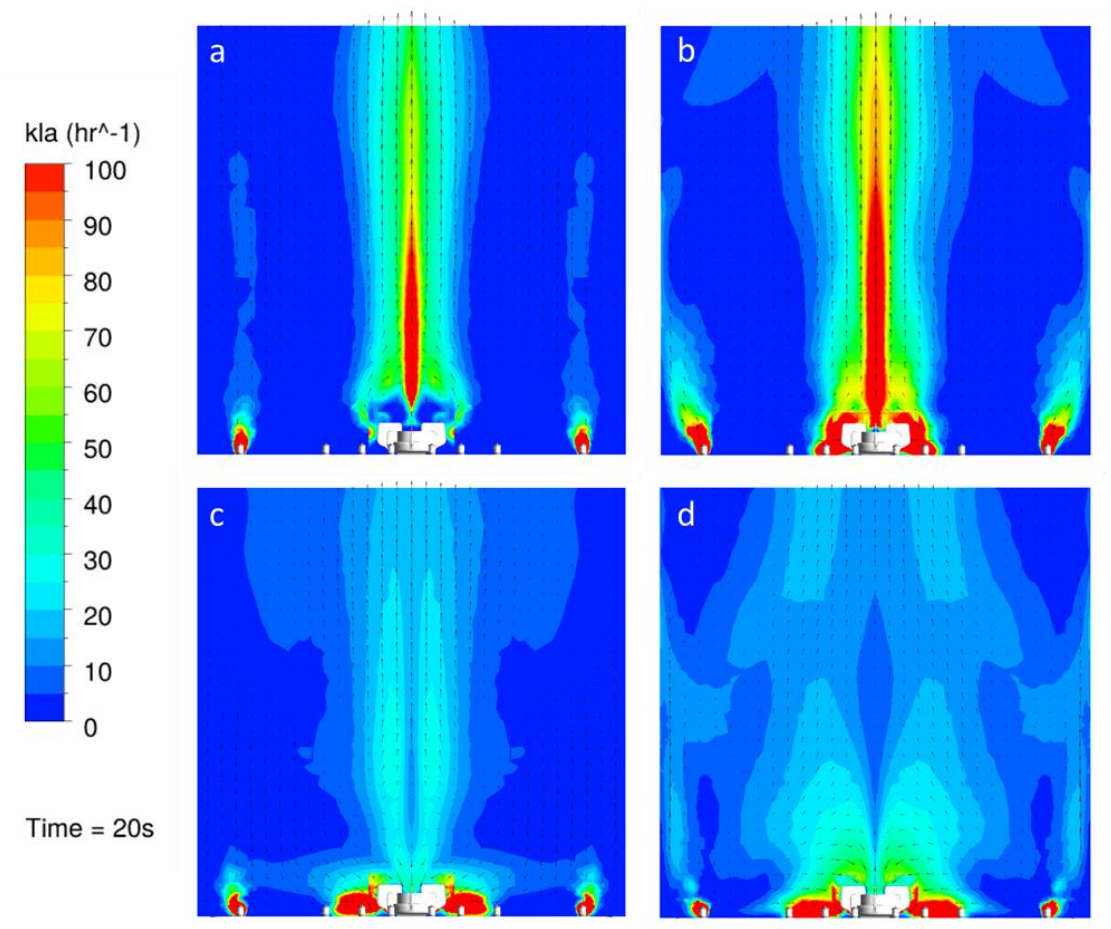

Fig. 3: Contours of $\mathrm{k}_{\mathrm{L}} \mathrm{a}$ and vectors of liquid velocity for a central cut-plane of a 1,000 L SUT vessel with 0.0675 vvm aeration rate and an impeller speed of a) 100 RPM, b) 200 RPM, c) 300 RPM, d) 400 RPM.

\subsection{Effect of Increasing Gas Flow-rate}

Changing the flow-rate of gas introduced to the tank via the spargers is one of the simplest adaptations that can be made in an attempt to increase the $\mathrm{k}_{\mathrm{L}} \mathrm{a}$ values. The results presented in section 4.1 are for a vvm of 0.0675 , however an aeration rate of $0.1 \mathrm{vvm}$ is also achievable with the existing setup. This has been modelled at stirring speeds of 400 and 500 RPM, as shown in Table 2 for the different models discussed previously. The mass transfer model presented for previous results, the eddy cell model, is highlighted in bold, although all models show a clear increase in volume-averaged $\mathrm{k}_{\mathrm{L}}$ a values between the two conditions. The effect of further increases is yet to be investigated.

Table 2: Volume-Averaged $\mathrm{k}_{\mathrm{L}}$ a values for different mass transfer modes at varying stirrer speeds and aeration rates for the 1,000 L tank.

\begin{tabular}{|l|c|c|c|c|c|c|c|}
\hline AERATION RATE (vVm) & \multicolumn{3}{|c|}{$\mathbf{0 . 0 6 7 5}$} & \multicolumn{2}{c|}{$\mathbf{0 . 1}$} \\
\hline IMPELLER SPEED (RPM) & $\mathbf{1 0 0}$ & $\mathbf{2 0 0}$ & $\mathbf{3 0 0}$ & $\mathbf{4 0 0}$ & $\mathbf{5 0 0}$ & $\mathbf{4 0 0}$ & $\mathbf{5 0 0}$ \\
\hline Penetration model & 10.1 & 15.9 & 19.6 & 22.8 & 23.6 & 35.6 & 32.6 \\
\hline Slip velocity model & 53.9 & 63.9 & 87.3 & 104 & 121 & 149 & 169 \\
\hline Eddy cell model & 3.59 & 5.64 & 6.95 & $\mathbf{8 . 0 9}$ & $\mathbf{8 . 3 7}$ & $\mathbf{1 2 . 6}$ & $\mathbf{1 1 . 5}$ \\
\hline Rigid model & 5.54 & 6.58 & 8.98 & 9.35 & 12.5 & 15.4 & 17.4 \\
\hline Surface renewal stretch model & 21.8 & 29.4 & 33.6 & 39.2 & 46.3 & 60.7 & 72.5 \\
\hline
\end{tabular}

\subsection{Effect of Reducing Tank Size}

In order to assess the impact of changing the tank size, the $200 \mathrm{~L}$ geometry was modelled with the same gas velocity as the low-vvm $1,000 \mathrm{~L}$ case $\left(0.1 \mathrm{~ms}^{-1}\right)$. The reduced tank size means that the vvm for the smaller geometry was significantly increased to 0.25. The result of this alteration can be seen in Figure 4 for an impeller speed of 400 RPM. The volumeaveraged $\mathrm{k}_{\mathrm{L}}$ a was modelled as $19.8 \mathrm{hr}^{-1}$, which is 2.45 times greater than the $1,000 \mathrm{~L}$ vessel at the same stirrer speed. Despite the smaller working volume, improving mass transfer means that a higher density of biomass can be produced within the 
available space. Furthermore, vessel size is thought to be less significant for SUT vessels as increases in production volume are easily achieved through numbering-up, where several reactors are used in parallel.

An analysis of the flow patterns in the $200 \mathrm{~L}$ tank at 400 RPM and $0.25 \mathrm{vvm}$ is made in Figure 5 by combining the gas volume fraction contour plots with vectors of liquid velocity. The results show a large central region where there is no gas present, and therefore no mass transfer occurring, accompanied by a strong downward flow of liquid. This shows that the recirculation currents are not strong enough to capture the gas phase when using only a single radial mixer. However, in the regions either side of this column there is a smaller recirculation loop seen, where the fluid travels up the side of the vessel and is drawn back towards the impeller. Figure $5 \mathrm{~b}$ shows that there is also significant recirculation in the horizontal plane at half of the filled height $(0.313 \mathrm{~m})$, suggesting better mixing throughout the majority of the vessel, excluding the central region.

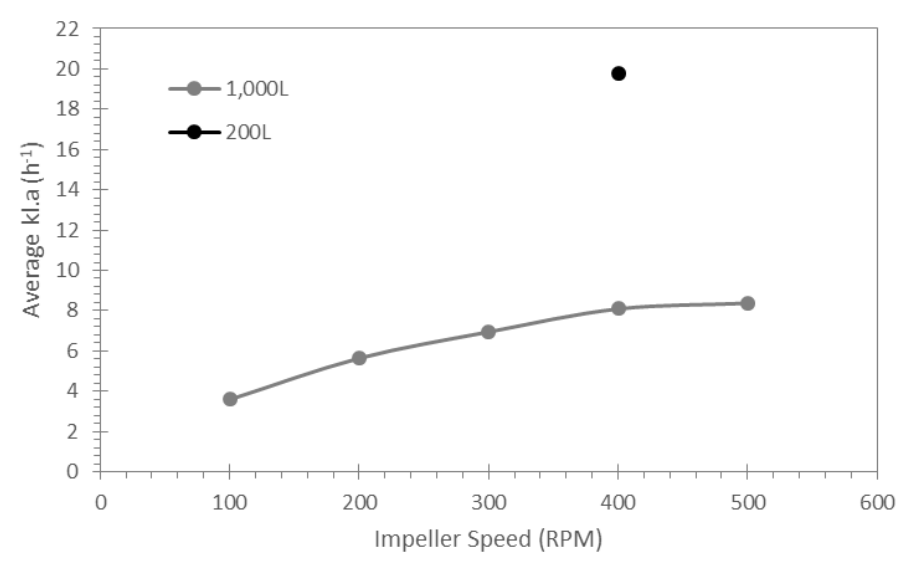

Fig. 4: Comparison of the volume-averaged $\mathrm{k}_{\mathrm{L}} \mathrm{a}$ values in the 1,000 and $200 \mathrm{~L}$ vessels using the eddy cell model.
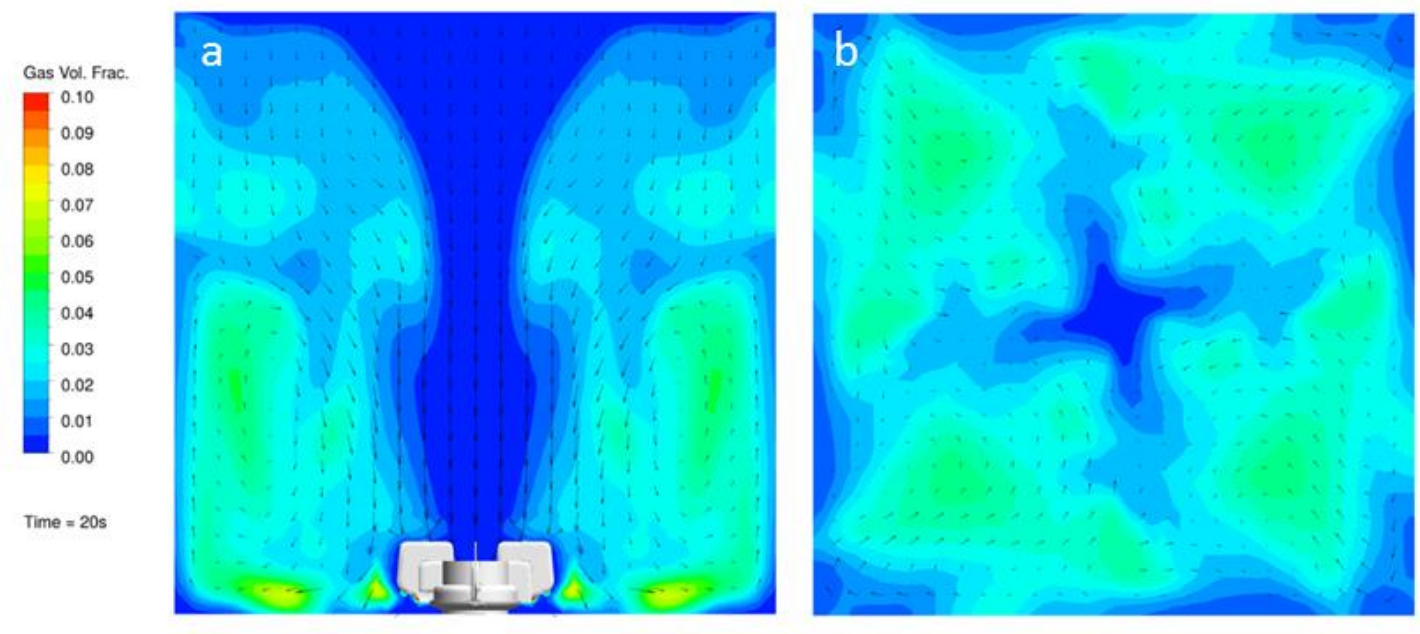

Fig. 5: Gas volume fraction contours and liquid velocity vector arrows in a $200 \mathrm{~L}$ vessel with a $0.25 \mathrm{vvm}$ aeration rate and $400 \mathrm{RPM}$ stirrer speed for a) vertical cut-plane through the centre of the tank and b) horizontal cut-plane at a height of $0.313 \mathrm{~m}$.

\section{Conclusions}

The mass transfer within a cubic SUT bioreactor has been investigated using CFD. Five different models for the mass transfer coefficient $\mathrm{k}_{\mathrm{L}}$ were identified from literature and compared. Four out of the five models (eddy cell, penetration, rigid and surface renewal stretch models) gave reasonably consistent values with the limited experimental data available, however the eddy cell model was concluded to be the most suitable to describe the mass transfer, and as the most conservative model also presents a 'worst case' value for design purposes. Between 400 and $500 \mathrm{RPM}$, volume-averaged $\mathrm{k}_{\mathrm{L} a}$ values calculated using the eddy cell model were seen to level off $(\mathrm{vvm}=0.0675)$ or decrease $(0.1 \mathrm{vvm})$, suggesting no additional benefit in 
increasing the stirrer speed above 400 RPM. This behaviour is not seen in some other models, where $k_{\mathrm{L}} \mathrm{a}$ continues to increase, suggesting further validation of model selection is needed in this region. Increasing the stirrer speed from 100 to 400 RPM showed an increase in volume-averaged $\mathrm{k}_{\mathrm{L}}$ a values, with radial gas dispersion only occurring above 300 RPM, and an optimum speed of 400 RPM identified. A much greater increase in $\mathrm{k}_{\mathrm{L}}$ a values was achieved by increasing the aeration rate from 0.0675 to $0.1 \mathrm{vvm}$, achieving approximately $50 \%$ improvement in volume-averaged values when modelled with either a 400 or 500 RPM stirrer speed. The biggest increase in $\mathrm{k}_{\mathrm{L}}$ a values was achieved by reducing the tank volume from 1,000 to $200 \mathrm{~L}$, attributed to a higher specific aeration rate and the greater recirculation modelled at 400 RPM, however strong downward currents produced a central column where no mass transfer was occurring.

\section{Acknowledgements}

The authors would like to acknowledge funding from Innovate UK (BioMOD), as well as the Centre for Sustainable Chemical Technologies (CSCT) at the University of Bath and the Engineering and Physical Sciences Research Council (EPSRC). Support and data supplied by the Centre for Process Innovation (CPI) is also gratefully acknowledged.

\section{References}

[1] R. Eibl, S. Kaiser, R. Lombriser, and D. Eibl, "Disposable bioreactors: The current state-of-the-art and recommended applications in biotechnology," Appl. Microbiol. Biotechnol., vol. 86, no. 1, pp. 41-49, 2010.

[2] R. Brecht, "Disposable Bioreactors: Maturation into Pharmaceutical Glycoprotein Manufacturing," in Disopsable Bioreactors, R. Eibl and D. Eibl, Eds. Heidelberg: Springer, 2009, pp. 1-32.

[3] A. G. Lopes, "Single-use in the biopharmaceutical industry: A review of current technology impact, challenges and limitations," Food Bioprod. Process., vol. 93, pp. 98-114, 2015.

[4] R. Eibl, S. Werner, and D. Eibl, "Disposable bioreactors for plant liquid cultures at litre-scale," Eng. Life Sci., vol. 9, no. 3, pp. 156-164, 2009.

[5] T. Kapp, J. Boehm, J. Chase, J. Craig, K. Davis, V. Gupta, S. A. Montgomery, and K. Ott, "Road Map to Implementation of Single-Use Systems," Bioprocess Int., vol. 8, no. 4, p. Supplement 10-19, 2010.

[6] B. Rawlings and H. Pora, "Environmental impact of single-use and reusable bioprocess systems," Bioprocess Int., vol. 7, no. 2, pp. 18-25, 2009.

[7] BPSA, "Guide to Disposal of Single-Use Bioprocess Systems," Bioprocess International, pp. 22-28, 2007.

[8] B. Andersson, R. Andersson, M. Mortensen, R. Sudiyo, and B. Van Wachem, Computational Fluid Dynamics for Engineers. Cambridge: Cambridge University Press, 2011.

[9] “ANSYS CFX-Solver Theory Guide.” ANSYS Inc., Canonsburg, 2013.

[10] A. R. Khopkar, A. R. Rammohan, V. V. Ranade, and M. P. Dudukovic, "Gas-liquid flow generated by a Rushton turbine in stirred vessel: CARPT/CT measurements and CFD simulations," Chem. Eng. Sci., vol. 60, no. 8-9, pp. 2215-2229, Apr. 2005.

[11] P. M. Doran, Bioprocess Engineering Principles. London: Elsevier, 1995.

[12] A. a Kulkarni, "Mass transfer in bubble column reactors: effect of bubble size distribution," Ind. Eng. Chem. Res., vol. 46, no. 7, pp. 2205-2211, 2007.

[13] P. Ranganathan and S. Sivaraman, "Investigations on hydrodynamics and mass transfer in gas-liquid stirred reactor using computational fluid dynamics," Chem. Eng. Sci., vol. 66, no. 14, pp. 3108-3124, 2011.

[14] R. Higbie, "The Rate of Absorbtion of a Pure Gas into a Still Liquid During Short Periods of Exposure," Trans. Am. Inst. Chem. Eng, vol. 31, pp. 365-389, 1935.

[15] J. C. Lamont and D. S. Scott, "An eddy cell model of mass transfer into the surface of a turbulent liquid," AIChE J., vol. 16, no. 4, pp. 513-519, Jul. 1970.

[16] S. S. Alves, C. I. Maia, and J. M. T. Vasconcelos, "Gas-liquid mass transfer coefficient in stirred tanks interpreted through bubble contamination kinetics," Chem. Eng. Process. Process Intensif., vol. 43, no. 7, pp. 823-830, 2004.

[17] B. Jajuee, A. Margaritis, D. Karamanev, and M. a. Bergougnou, "Application of surface-renewal-stretch model for interface mass transfer,” Chem. Eng. Sci., vol. 61, no. 12, pp. 3917-3929, 2006. 